\title{
Erratum: Feynman-Schwinger representation approach to nonperturbative physics [Phys. Rev. C 60, 055210 (1999)]
}

Çetin Şavklı, John Tjon, and Franz Gross

(Published 16 May 2000)

PACS number(s): 11.15.Tk, 11.10.St, 99.10.+g

We are indebted to R. Rosenfelder and A. W. Schreiber for calling attention to some errors in our paper and for raising the issue of the instability of $\chi^{2} \phi$ theories [1]. The following errors were found in the manuscript:

(1) The critical coupling constant in Fig. 3 [and in the text following Eq. (2.27)] is $0.402(\mathrm{GeV})^{3}$, instead of $0.343(\mathrm{GeV})^{2}$. The critical mass is $1.45 \mathrm{GeV}$ instead of $1.49 \mathrm{GeV}$. Figure 3 is correct.

(2) In Eqs. (2.7) and (3.3) a factor of $\operatorname{det} S$ should appear in the right-hand side (rhs) under the integral instead of (detS) ${ }^{-1}$. Since $\operatorname{det} S=1$ in the quenched approximation, this does not affect any of the results in the paper.

(3) The rhs of the second form of Eq. (3.38) should be divided by $p^{2}$ (this was a writing error; the numerical results are unchanged).

Rosenfelder and Schreiber [1] also called our attention to one of their papers [2] and raised the issue of whether or not our calculations of masses should show the instabilities known to accompany theories of the $\phi^{3}$ type [3]. The calculations presented in our paper are stable (at least for small coupling constants; we will show in a forthcoming paper that instabilities arise for large coupling constants). We suspect this is because we use the quenched approximation, with results parallel to those obtained from bound state equations. (Baym's original proof [3] explicitly exploits the possibility that $\chi \bar{\chi}$ pairs may be produced from the vacuum, and hence no proof exists for the quenched approximation.) In any case we agree with Rosenfelder and Schreiber that the stability issue deserves further study.

[1] R. Rosenfelder (private communication).

[2] R. Rosenfelder and A. W. Schreiber, Phys. Rev. D 53, 3337 (1996).

[3] G. Baym, Phys. Rev. 117, 886 (1960). 